\title{
Das neue Wahlgesetz im Test der Bundestagswahl 2013
}

\author{
Joachim Behnke
}

\section{Das neue Wahlrecht: inkonsistent, widersprüchlich, ineffektiv}

Die Bundestagswahl im September 2013 fand nach einem neuen Wahlgesetz statt, das erst kurz zuvor von den Fraktionen der CDU/CSU, SPD, FDP und der Grünen verabschiedet worden war. Es war das Ergebnis einer Wahlrechtsdebatte, die mit der Häufung der Überhangmandate nach der Wiedervereinigung begonnen hatte und mit dem Urteil des Bundesverfassungsgerichts vom 3. Juli 2008 zum negativen Stimmgewicht noch einmal an Dringlichkeit gewonnen hatte, weil der Gesetzgeber nun sogar dazu verpflichtet worden war, das bestehende Wahlgesetz zu überarbeiten. Dafür gewährte das BVerfG - in der Annahme, damit keine leicht zu bewältigende Aufgabe zu stellen - eine großzügige Frist bis zum 30. Juni 2011.

Die ersten Entwürfe für ein neues Gesetz lagen im Sommer 2011 vor. ${ }^{1}$ Inhaltlich unterschieden sich dabei die von den Parteien verfolgten Lösungswege, um das negative Stimmgewicht zu beheben, erheblich. Da dieser Effekt im alten Wahlgesetz von 2008 über die Entstehung von Überhangmandaten vermittelt auftrat, gab es zwei prinzipiell verschiedene Ansätze: Der erste sah vor, das Problem des negativen Stimmgewichts durch die Abschaffung beziehungsweise Neutralisierung der Überhangmandate gewissermaßen „en passant“ lösen zu wollen. Diesen Weg verfolgten die SPD, die Grünen und die Linke. Der Entwurf der Grünen sah vor, die Überhangmandate einer Partei durch die Streichung von Listenmandaten in anderen Bundesländern zu kompensieren; die SPD sprach sich für ein Ausgleichsmodell aus, und der Gesetzentwurf der Linken verband beide Modelle geschickt miteinander. Der zweite Ansatz, den die CDU/CSU und die FDP verfolgten, sollte das Problem des negativen Stimmgewichts unabhängig von dem der Überhangmandate lösen. Dafür wollte man die Verrechnung zwischen den Landeslisten unterbinden, indem die Sitze in einem ersten Schritt nicht auf die Parteien, sondern entsprechend den Wählerstimmen auf die Bundesländer verteilt wurden. Im Dezember 2011 verabschiedete die Regierungskoalition aus CDU, CSU und FDP auf der Basis dieses Entwurfs das neue Wahlgesetz.

Dies wurde allerdings mit dem BVerfG-Urteil vom 25. Juli 2012 für verfassungswidrig erklärt, unter anderem, weil auch das neue Wahlgesetz immer noch das negative Stimmgewicht zuließ und wegen der fehlenden Behandlung des Problems der Überhangmandate. ${ }^{2}$ Nach diesem Urteil verständigten sich die Fraktionen darauf zu versuchen, das nächste Wahlgesetz im parteienübergreifenden Konsens zu verabschieden. Das Ergebnis dieses weitgehenden Konsenses, dem sich alle bis auf die Linke anschlossen, war das neue Wahlgesetz, das am 3. Mai 2013 verabschiedet wurde.

1 Vgl. BT-Drucksache 17/6290 (Entwurf der CDU, CSU und FDP vom 28. Juni 2011), BTDrucksache 17/5895 (Entwurf der SPD vom 24. Mai 2011), BT-Drucksache 17/5896 (Entwurf der Linken vom 25. Mai 2011) und BT-Drucksache 17/4694 (Entwurf der Grünen vom 9. Februar 2011).

2 Vgl. BVerfG, 2 BvF 3/11 vom 25. Juli 2012, Absatz-Nr. (1 - 164), http://www.bverfg.de/entscheidungen/fs20120725_2bvf000311.html (Abruf am 23. Januar 2014). 
Das neue Gesetz stellt in einigen relevanten Punkten eine Verbesserung nicht nur gegenüber dem so offensichtlich fehlerhaften Wahlgesetz von 2011, sondern auch gegenüber dem zuvor geltenden von 2008 dar. Der darin enthaltene Ausgleich garantiert die Geltung der Erfolgswertgleichheit und beseitigt die wesentlichen durch Überhangmandate bedingten Probleme, da diese keine Veränderungen der relativen Stärkeverhältnisse mehr bewirken können. Damit ist vor allem die größte demokratietheoretische Gefahr gebannt, die vom alten Gesetz ausging, nämlich die der Umkehrung von Mehrheitsverhältnissen.

Dennoch sind auch einige Schwächen deutlich zu erkennen. ${ }^{3}$ Eine besteht allein schon in der Unverständlichkeit des neuen Gesetzes, das in dieser Beziehung neue Maßstäbe setzt und der Forderung des Verfassungsgerichts im Urteil von 2008, ein neues Gesetz auf eine „normenklare und verständliche Grundlage zu stellen“, in keiner Weise gerecht wird. Der Verfassungsrechtler Hans Meyer, der die Wahlrechtsdebatte über Jahrzehnte hinweg wie kein anderer geprägt hat, spricht von einem „Muster grob missratener Gesetzgebungskunst“ " Florian Grotz von einem „massiven Verständlichkeitsproblem "5; Friedrich Pukelsheim und Matthias Rossi halten eine Reform auch „angesichts insbesondere der sprachlich missglückten Formulierung des Gesetzestextes "6 für notwendig. Diese Unverständlichkeit ist aus demokratietheoretischer Sicht insofern höchst bedauerlich und problematisch, als sie zur Folge hat, dass der Bürger den zentralen Akt der Demokratie - die Übertragung seiner Souveränitätsrechte an die von ihm gewählten Repräsentanten - auf eine Weise vollziehen muss, die für ihn selbst nicht nachvollziehbar ist. Die Unverständlichkeit, wie ein bestimmter Effekt zustande kommt, besagt allerdings noch nicht, dass der Effekt selbst ebenfalls problematisch ist. Hätte man sich nur zwischen einem unverständlichen, aber höchst gerechten Wahlsystem und einem verständlichen, aber sehr ungerechten Wahlsystem zu entscheiden, so gäbe es vermutlich gute Gründe, hier einen „Trade off“ zu Gunsten der Gerechtigkeit auf Kosten der Verständlichkeit vorzunehmen. Die Unverständlichkeit mag dann der Preis für ein Wahlrecht sein, das ansonsten alle vernünftigen Kriterien an ein ,gutes" Wahlsystem erfüllt, beziehungsweise im schlimmsten Fall sogar der unvermeidliche Preis, der angesichts der einzuhaltenden Restriktionen notwendigerweise zu entrichten war. Doch diese Sichtweise ist nicht stichhaltig: Dieselben Gestaltungsentscheidungen, die die Kompliziertheit und Unverständlichkeit bedingen, führen auch zu Effekten, die klar gegen gewisse Anforderungen verstoßen, die man vernünftigerweise an ein gelungenes Design eines Wahlsystems stellen sollte. Das neue Wahlrecht ist zwar nicht ungerecht, aber es ist inkonsistent, in sich widersprüchlich und extrem ineffizient. All dies

3 Vorwiegend kritisch zum neuen Gesetz daher unter anderem Niels Dehmel / Eckhard Jesse, Das neue Wahlgesetz zur Bundestagswahl 2013. Eine Reform der Reform der Reform ist unvermeidlich, in: ZParl, 44. Jg. (2013), H. 1, S. 201 - 213; Joachim Behnke, Das neue Wahlgesetz, sicherlich nicht das letzte, in: Recht und Politik, 49. Jg. (2013), H. 1, S. 1 - 10; Hans Meyer, Das Bundestagswahlrecht 2013, in: Der Bürger im Staat, 63. Jg. (2013), H. 3, S. 208 - 217; Florian Grotz, Happy End oder endloses Drama? Die Reform des Bundestagswahlsystems, in: Eckhard Jesse / Roland Sturm (Hrsg.), Bilanz der Bundestagswahl 2013, Voraussetzungen, Ergebnisse, Folgen, Baden-Baden (im Erscheinen); Friedrich Pukelsheim / Matthias Rossi, Imperfektes Wahlrecht, in: Zeitschrift für Gesetzgebung, 28. Jg. (2013), H. 3, S. 209 - 226; eher positiv hingegen Gerd Strohmeier, Kein perfektes Wahlsystem, aber ein guter Kompromiss - unter schwierigen Rahmenbedingungen, in: Zeitschrift für Politik, 60. Jg. (2013), H. 2, S. $144-161$.

6 Friedrich Pukelsheim / Matthias Rossi, a.a.O. (Fn. 3), S. 222. 
lässt sich leicht belegen, wenn man die Bundestagswahl 2013 als den ersten empirischen Test betrachtet, den das neue Gesetz zu bestehen hatte.

\section{Die Sitzverteilung nach dem neuen Wahlgesetz}

Nach dem neuen Wahlgesetz erfolgt die Verteilung der Sitze mehrstufig. Auf der ersten Stufe werden zuerst die Sitze zur Bildung von Kontingenten proportional nach der Bevölkerungszahl auf die einzelnen Bundesländer verteilt. Das hierbei verwandte Berechnungsverfahren ist das nach Sainte-Laguë (vgl. Tabelle 1).

\begin{tabular}{|c|c|c|c|c|c|c|}
\hline \multicolumn{7}{|c|}{$\begin{array}{l}\text { Tabelle 1: Verteilung der Sitze auf die Bundesländer nach Bevölkerungszahl bei der Bundestags- } \\
\text { wahl } 2013\end{array}$} \\
\hline Bundesland & Bevölkerung & $\begin{array}{l}\text { Sitze nach } \\
\text { Bevölke- } \\
\text { rung }\end{array}$ & $\begin{array}{l}\text { Zweit- } \\
\text { stimmen }\end{array}$ & $\begin{array}{c}\text { Sitze nach } \\
\text { Zweitstim- } \\
\text { men }\end{array}$ & $\begin{array}{l}\text { Über- } \\
\text { repräsen- } \\
\text { tation }\end{array}$ & $\begin{array}{c}\text { Zweit- } \\
\text { stimmen/ } \\
\text { Bevölkerung } \\
\left({ }^{*} 100\right) \\
\end{array}$ \\
\hline Baden-Württemberg & 9.482 .902 & 76 & 4.632 .780 & 75 & 1,013 & 48,85 \\
\hline Bayern & 11.353 .264 & 92 & 5.359 .316 & 87 & 1,057 & 47,21 \\
\hline Berlin & 3.025 .288 & 24 & 1.499 .274 & 24 & 1,000 & 49,56 \\
\hline Brandenburg & 2.418 .267 & 19 & 1.180 .269 & 19 & 1,000 & 48,81 \\
\hline Bremen & 575.805 & 5 & 286.961 & 5 & 1,000 & 49,84 \\
\hline Hamburg & 1.559 .655 & 13 & 765.951 & 12 & 1,083 & 49,11 \\
\hline Hessen & 5.388 .350 & 43 & 2.641 .689 & 43 & 1,000 & 49,03 \\
\hline $\begin{array}{l}\text { Mecklenburg- } \\
\text { Vorpommern }\end{array}$ & 1.585 .032 & 13 & 748.066 & 12 & 1,083 & 47,20 \\
\hline Niedersachsen & 7.354 .892 & 59 & 3.911 .433 & 63 & 0,937 & 53,18 \\
\hline Nordrhein-Westfalen & 15.895 .182 & 128 & 8.148 .412 & 132 & 0,970 & 51,26 \\
\hline Rheinland-Pfalz & 3.672 .888 & 30 & 1.857.275 & 30 & 1,000 & 50,57 \\
\hline Saarland & 919.402 & 7 & 475.003 & 8 & 0,875 & 51,66 \\
\hline Sachsen & 4.005 .278 & 32 & 1.916 .381 & 31 & 1,032 & 47,85 \\
\hline Sachsen-Anhalt & 2.247 .673 & 18 & 1.029 .689 & 17 & 1,059 & 45,81 \\
\hline Schleswig-Holstein & 2.686 .085 & 22 & 1.389 .795 & 23 & 0,957 & 51,74 \\
\hline Thüringen & 2.154 .202 & 17 & 1.025 .123 & 17 & 1,000 & 47,59 \\
\hline Bund & 74.324 .165 & 598 & 36.867 .417 & 598 & 1,000 & 49,60 \\
\hline
\end{tabular}

Bei der Bundestagswahl 2013 entfielen demnach auf Bayern mit seiner Bevölkerung von etwas mehr als elf Millionen zum Beispiel 92 Sitze, auf Baden-Württemberg mit einer Bevölkerungszahl von circa neuneinhalb Millionen 76 usw. Obwohl die Sitze letztendlich entsprechend der abgegebenen Wählerstimmen verteilt werden müssen, da jeder Sitzanspruch nur durch Wählerstimmen begründet sein kann, erfolgt die Verteilung auf der ersten Stufe nach dem eigentlich irrelevanten Kriterium der Bevölkerungszahl. ${ }^{7}$ Die Ver-

7 Die „Irrelevanz“ bezieht sich dabei auf zwei verschiedene Aspekte, der eine ist rein technischer Art, der zweite substanzieller. Der technische Aspekt bezieht sich darauf, dass sich die Zuteilung 
wendung dieses Verteilungskriteriums, das der Logik der letztendlichen Sitzverteilung wesensfremd ist, hat weitreichende Konsequenzen. Hätte man die 598 Sitze zum Beispiel direkt entsprechend der Zweitstimmen, die für eine der zuteilungsberechtigten Parteien abgegeben wurden, verteilt, hätte sich die in der fünften Spalte von Tabelle 1 aufgeführte Verteilung ergeben. Wie man sieht, dürften auf Bayern eigentlich nur 87 Sitze entfallen und nicht die 92, die ihm nach dem neuen Wahlgesetz zuerkannt werden. Das Landessitzkontingent für Bayern ist auf der ersten Stufe also zu groß, das heißt alle bayerischen Landeslisten der Parteien sind dort überrepräsentiert. Die Überrepräsentation eines Ländersitzkontingents fällt dabei, abgesehen von Rundungseffekten, desto höher aus, je niedriger der Anteil der zu verrechnenden Zweitstimmen an der Bevölkerungszahl ist.

Als nächstes werden die Sitze des Kontingents auf die Landeslisten der Parteien im jeweiligen Land verteilt und zwar entsprechend den Zweitstimmen, die diese erhalten haben. Auch hier findet wieder das Zuteilungsverfahren nach Sainte-Laguë Verwendung. Wie bisher auch, nehmen daran nur diejenigen Parteien teil, die entweder bundesweit fünf Prozent der Zweitstimmen oder zumindest drei Direktmandate erhalten haben. Nach diesem Selektionskriterium werden nur noch CDU, CSU, SPD, Grüne und Linke bei der Verteilung der Sitze bedacht. Es ergaben sich so die in Tabelle 2 aufgeführten Zahlen, die jeweils unter dem Etikett „PR“ (für Proporzmandate) zu finden sind. Von den 92 Sitzen für Bayern entfallen zum Beispiel nach Proporz 23 auf die SPD, 56 auf die CSU, neun auf die Grünen und vier auf die Linke. Diese Verteilung wird im neuen Wahlgesetz als „erste Verteilung“ bezeichnet ( $\$ 6 \mathrm{Ab}$ satz 2 BWG). Zusätzlich zu diesen Proporzmandaten ist in der Tabelle 2 angegeben, wie viele Direktmandate die einzelnen Parteien in den jeweiligen Bundesländern gewonnen haben.

In einigen Bundesländern konnte eine Partei - 2013 betrifft dies nur die CDU - mehr Direktmandate erzielen, als ihr entsprechend ihrem Zweitstimmenanteil in diesem Bundesland eigentlich zustehen würde. Diese Mandate entsprechen in gewisser Weise „Überhangmandaten“. Allerdings ist dieser Ausdruck hier nur mit Vorsicht zu gebrauchen, da diese „Überhangmandate“ nicht jenen entsprechen, die zum Beispiel im alten Wahlgesetz von 2008 auftraten. Um Missverständnisse zu vermeiden, ist es daher sinnvoll, sie mit einem speziellen Begriff zu versehen. Da sie auf der ersten Verteilungsstufe entstehen, sollen sie als „Quasi-Überhangmandate der ersten Stufe“ oder einfach nur als „Quasi-Überhangmandate" bezeichnet werden. Davon gab es 2013 insgesamt vier, jeweils eines in Brandenburg, Saarland, Sachsen-Anhalt und Thüringen.

der Sitze im neuen Wahlgesetz auf der letzten Stufe der Verteilung, das heißt genau genommen ab dem Ausgleich, ausschließlich an den Zweitstimmen orientiert. Die Bevölkerungszahlen werden dadurch als Verteilungskriterium am Schluss irrelevant gemacht. Der substanzielle Aspekt bezieht sich auf den Charakter des Bundestags als „unitarisches Vertretungsorgan des Bundesvolks“ (BVerfG, 2 BvC 1/07 vom 3. Juli 2008, Absatz-Nr. 120). Im Urteil von 2008 spricht das BVerfG auch von „historisch gewachsenen Traditionen“, nach denen „föderale Belange nicht das System des Bundeswahlgesetzes bestimmen“ (Absatz-Nr. 121), auch wenn die Ausgestaltung einzelner technischer Elemente wie der Landeslisten diesen föderalen Traditionen geschuldet ist. Die Bürger wählen bei der Bundestagswahl daher als Deutsche und nicht als Bayern, Sachsen oder Angehörige anderer Bundesländer. Eine fixe Zuteilung von Ländersitzkontingenten aufgrund der Bevölkerungszahlen hätte aber zur Folge, dass der Erfolgswert von Wählern verschiedener Bundesländer in Abhängigkeit von der Wahlbeteiligung variieren würde. Es wäre aber schwer vermittelbar, warum ein bayerischer Deutscher ein höheres Stimmgewicht erhalten sollte, weil besonders viele andere bayerische Deutsche von ihrem Wahlrecht nicht Gebrauch gemacht oder für eine Partei gestimmt haben, die nicht ins Parlament gelangt. 


\begin{tabular}{|c|c|c|c|c|c|c|c|c|c|c|c|c|}
\hline & \multicolumn{2}{|c|}{ SPD } & \multicolumn{2}{|c|}{$\mathrm{CDU}$} & \multicolumn{2}{|c|}{$\mathrm{CSU}$} & \multicolumn{2}{|c|}{ Grüne } & \multicolumn{2}{|c|}{ Linke } & \multicolumn{2}{|c|}{ alle } \\
\hline & PR & $\mathrm{DM}$ & PR & DM & PR & $\mathrm{DM}$ & PR & $\mathrm{DM}$ & PR & DM & PR & DM \\
\hline Baden-Württemberg & 19 & 0 & 43 & 38 & 0 & 0 & 10 & 0 & 4 & 0 & 76 & 38 \\
\hline Bayern & 23 & 0 & 0 & 0 & 56 & 45 & 9 & 0 & 4 & 0 & 92 & 45 \\
\hline Berlin & 7 & 2 & 8 & 5 & 0 & 0 & 4 & 1 & 5 & 4 & 24 & 12 \\
\hline Brandenburg & 5 & 1 & 8 & 9 & 0 & 0 & 1 & 0 & 5 & 0 & 19 & 10 \\
\hline Bremen & 2 & 2 & 1 & 0 & 0 & 0 & 1 & 0 & 1 & 0 & 5 & 2 \\
\hline Hamburg & 5 & 5 & 5 & 1 & 0 & 0 & 2 & 0 & 1 & 0 & 13 & 6 \\
\hline Hessen & 15 & 5 & 20 & 17 & 0 & 0 & 5 & 0 & 3 & 0 & 43 & 22 \\
\hline $\begin{array}{l}\text { Mecklenburg- } \\
\text { Vorpommern }\end{array}$ & 3 & 0 & 6 & 6 & 0 & 0 & 1 & 0 & 3 & 0 & 13 & 6 \\
\hline Niedersachsen & 22 & 13 & 28 & 17 & 0 & 0 & 6 & 0 & 3 & 0 & 59 & 30 \\
\hline Nordrhein-Westfalen & 48 & 27 & 59 & 37 & 0 & 0 & 12 & 0 & 9 & 0 & 128 & 64 \\
\hline Rheinland-Pfalz & 10 & 1 & 15 & 14 & 0 & 0 & 3 & 0 & 2 & 0 & 30 & 15 \\
\hline Saarland & 3 & 0 & 3 & 4 & 0 & 0 & 0 & 0 & 1 & 0 & 7 & 4 \\
\hline Sachsen & 6 & 0 & 16 & 16 & 0 & 0 & 2 & 0 & 8 & 0 & 32 & 16 \\
\hline Sachsen-Anhalt & 4 & 0 & 8 & 9 & 0 & 0 & 1 & 0 & 5 & 0 & 18 & 9 \\
\hline Schleswig-Holstein & 8 & 2 & 10 & 9 & 0 & 0 & 3 & 0 & 1 & 0 & 22 & 11 \\
\hline Thüringen & 3 & 0 & 8 & 9 & 0 & 0 & 1 & 0 & 5 & 0 & 17 & 9 \\
\hline Bund & 183 & 58 & 238 & 191 & 56 & 45 & 61 & 1 & 60 & 4 & 598 & 299 \\
\hline $\begin{array}{l}\text { Bund Grundvertei- } \\
\text { lung }\end{array}$ & & 33 & & 42 & & 6 & & 1 & & & & \\
\hline
\end{tabular}

Als Basis für das zu Beginn der zweiten Stufe folgende Ausgleichsverfahren werden nun für jede Partei die Sitzzahlen berechnet, die ihr in den einzelnen Bundesländern zustehen. Diese Zahlen sollen hier als „Sitzansprüche“ bezeichnet werden. ${ }^{8}$ Der Sitzanspruch einer Partei in einem bestimmten Bundesland entspricht dem Maximum aus der Zahl der Proporzmandate und der Zahl der Direktmandate, die der Partei in diesem Bundesland zustehen. Er ist also üblicherweise gleich den Proporzmandaten, nur in Fällen, in denen QuasiÜberhangmandate auftreten, wird er um deren Anzahl erhöht. Die Verteilung der Sitzansprüche wird im Folgenden auch als Grundverteilung bezeichnet, da sie den Abschluss der Sitzverteilung auf der ersten Stufe und die entscheidende Grundlage für die Verteilung auf der zweiten Stufe darstellt. Die Grundverteilung ist nicht mit der „ersten Verteilung“ im Sinne des neuen Wahlgesetzes zu verwechseln; sie setzt sich zusammen aus der „ersten Verteilung“ und den Quasi-Überhangmandaten. Der Gesamtsitzanspruch für eine Partei ist die Summe ihrer Sitzansprüche in den Bundesländern. Diese Zahlen sind in der letzten Zeile von Tabelle 2 abgebildet.

Die zweite Stufe des Verfahrens beginnt mit dem Ausgleich. Damit soll der bundesweite Proporz zwischen den Parteien in Bezug auf ihre Zweitstimmenergebnisse hergestellt werden. Dazu wird die Gesamtzahl der Sitze so lange erhöht, bis die (bundesweiten) Gesamt-

8 Auf die mögliche Problematik des Begriffs „Anspruch“ wird noch zurückzukommen sein. 
sitzansprüche jeder Partei, wie sie in der Grundverteilung aufgezeigt sind, durch die Zweitstimmenanteile der Parteien abgedeckt sind. Die Sitzzahl wird also solange erhöht, bis jeder Partei entsprechend ihren Zweitstimmen mindestens so viele Sitze zustehen, wie sie in der Grundverteilung insgesamt erhalten hat. Der Ausgleich orientiert sich daher an der Partei, die in der Grundverteilung am stärksten überrepräsentiert ist. Entgegen den spontanen Vermutungen muss dies jedoch keineswegs die Partei sein, die die meisten Überhangmandate erhält. Wie in Tabelle 3 zu sehen, ist die nach der ersten Stufe am stärksten überrepräsentierte Partei nämlich die CSU und nicht die CDU. Mit 8,8 Prozent der zu verrechnenden Zweitstimmen kommt die CSU auf 9,3 Prozent der 602 Sitze, die insgesamt auf der ersten Stufe verteilt werden. Damit ist sie um ungefähr 5,7 Prozent überrepräsentiert. Die CDU ist hingegen sogar trotz der Quasi-Überhangmandate immer noch leicht unterrepräsentiert.

\begin{tabular}{|c|c|c|c|c|c|c|}
\hline \multirow[t]{2}{*}{ Tabelle 3: } & $\begin{array}{l}\text { ng der Zwei } \\
\text { erteilung }\end{array}$ & timmen der & teilungsber & btigten Pa & ien und Si & ablen in der \\
\hline & SPD & $\mathrm{CDU}$ & CSU & Grüne & Linke & alle \\
\hline $\begin{array}{l}\text { Zweitstimmen } \\
\text { absolut } \\
\text { in Prozent }\end{array}$ & $\begin{array}{r}11.252 .215 \\
30.52\end{array}$ & $\begin{array}{r}14.921 .877 \\
40.47\end{array}$ & $\begin{array}{r}3.243 .569 \\
8.8\end{array}$ & $\begin{array}{r}3.694 .057 \\
10.02\end{array}$ & $\begin{array}{r}3.755 .699 \\
1019\end{array}$ & $\begin{array}{r}36.867 .417 \\
100\end{array}$ \\
\hline $\begin{array}{l}\text { Sitze } \\
\text { absolut } \\
\text { in Prozent }\end{array}$ & $\begin{array}{r}183 \\
30,4\end{array}$ & $\begin{array}{r}242 \\
40,2\end{array}$ & $\begin{array}{r}56 \\
9,3\end{array}$ & $\begin{array}{r}61 \\
10,13\end{array}$ & $\begin{array}{r}60 \\
9,97\end{array}$ & $\begin{array}{l}602 \\
100\end{array}$ \\
\hline $\begin{array}{l}\text { Verhältnis Sitzzahl } \\
\text { zu Zweitstimmen }\end{array}$ & 0,996 & 0,993 & 1,057 & 1,011 & 0,978 & 1 \\
\hline
\end{tabular}

Festzuhalten ist daher, dass der Ausgleich seinen ursprünglichen Zweck, die durch die Überhangmandate entstandenen Verzerrungen des Proporzes zu neutralisieren, nicht erreicht. „Die Geister des Ausgleichsmechanismus, die man rief, wird man nicht so leicht los, und der Ausgleichsmechanismus sucht sich dann gewissermaßen jenseits seines ursprünglichen Zwecks ein anderes Betätigungsfeld. " ${ }^{\text {9 }}$ Die Orientierung der Berechnung der Landessitzkontingente an den Bevölkerungszahlen hat zur Folge, dass es auch unabhängig von Überhangmandaten zu nicht unerheblichen Verzerrungen des Proporzes kommen kann. Der Ausgleich bezieht sich auf die entstandene Proporzverzerrung und nicht auf die Ursache ihrer Entstehung. Florian Grotz spricht daher von "unechten Ausgleichsmandaten“10. Der Ausdruck ist aber nicht ganz zutreffend, da der Ausgleich ja ein „echter“ ist und seine Funktion, die Neutralisierung von Proporzverzerrungen, auch sehr wohl erfüllt; er tut dies aber eben nicht in Verfolgung des Zwecks, an den der Gesetzgeber bei der Schaffung des Ausgleichs ursprünglich gedacht haben dürfte, nämlich Überhangmandate auszugleichen. Dies ist insofern problematisch, als die Überhangmandate im alten Wahlgesetz eine

9 Joachim Behnke, Das neue Wahlgesetz - oder: Was lange währt, wird nicht unbedingt gut, in: Reimut Zohlnhöfer / Thomas Saalfeld (Hrsg.), Politik im Schatten der Krise. Eine Bilanz der Regierung Merkel 2009-2013, Wiesbaden (im Erscheinen).

10 Florian Grotz, a.a.O. (Fn. 3). 
zwingende Folge der personalisierten Verhältniswahl, wie sie im alten Wahlgesetz umgesetzt wurde, darstellten. ${ }^{11}$ Will man die dadurch entstandene Verletzung des Prinzips der Erfolgswertgleichheit durch Ausgleichsmandate "heilen“, dann sind diese Ausgleichsmandate genauso zwingend notwendig wie die Entstehung der Überhangmandate zwingend aus der Verknüpfung von Personalwahl und Verhältniswahl folgt. ${ }^{12}$ Damit erst kann die Schaffung zusätzlicher Mandate in Form von Ausgleichsmandaten überhaupt begründet werden. Ein Ausgleich für Mandate, die ohne zwingenden Grund entstehen, kann dementsprechend nicht schlüssig begründet werden. Es handelt sich dann nicht um einen „unechten“, sondern um einen „überflüssigen Ausgleich“.

Dieser orientierte sich 2013 also an der CSU, die nach dem Proporzprinzip nur auf 53 Sitze Anspruch hätte, aber in der Grundverteilung 56 Sitze erhält. ${ }^{13}$ Diese würden ihr bei einer proportionalen (bundesweiten) Zuteilung der Sitze aufgrund ihrer Zweitstimmen aber erst dann zustehen, wenn man nicht 598, sondern 631 verteilte. Ausgehend von dieser Sitzzahl des Parlaments verläuft nun die Sitzverteilung weitgehend wie beim alten Wahlgesetz. Zuerst wird die Oberverteilung ermittelt, das heißt die 631 Sitze werden entsprechend dem bundesweiten Zweitstimmenanteil auf die Parteien verteilt. Abgesehen von der CSU erhöht sich durch die Ausgleichsmandate für alle Parteien die Sitzzahl. Die SPD erhält gegenüber der Grundverteilung zehn zusätzliche Mandate, die CDU 13, die Grünen zwei und die Linke vier. Das Sitzkontingent einer Partei aus der Oberverteilung wird nun in der Unterverteilung entsprechend den Zweitstimmen auf die Landeslisten der Partei verteilt. In jedem Bundesland erhält die Partei aber mindestens so viele Sitze, wie sie dort Direktmandate errungen hat. Dies entspricht der Logik so genannter Verrechnungs- oder Kompensationsmodelle, in denen die Überhangmandate einer Partei mit Listenmandaten verrechnet werden. ${ }^{14}$ Auch die Gesetzentwürfe der Grünen und der Linken von 2011 enthielten dieses Element. Gemäß diesem Verfahren erhält man als Endergebnis die in Tabelle 4 dargestellte Sitzverteilung. In Klammern sind die Sitzzahlen angegeben, die entstünden, wenn alle 631 Sitze streng nach Proporz anhand der Zweitstimmen vergeben würden.

Ausgehend von $631 \mathrm{zu}$ verteilenden Sitzen fallen also weiterhin interne Überhangmandate an, eines in Brandenburg, eines in Sachsen-Anhalt und eines in Thüringen. Diese drei werden mit Listenmandaten der CDU in den Ländern verrechnet, in denen sie keine Über-

11 Nur weil die Entstehung der Überhangmandate zwingend aus der Konstruktion der personalisierten Verhältniswahl entsteht, heißt dies nicht, dass daher notwendigerweise eine Verletzung des Prinzips der Erfolgswertgleichheit hingenommen werden muss, da diese ja durch verschiedene Maßnahmen wie Ausgleichsmandate oder Verrechnung der Überhangmandate mit Listenmandaten sehr wohl "geheilt" werden kann, ohne die personalisierte Verhältniswahl in irgendeiner Form anzutasten.

12 Dies heißt allerdings keineswegs, dass der Proporz nur durch Ausgleichsmandate wieder hergestellt werden kann. Wenn man sich aber einmal entschieden hat, den Proporz durch Ausgleichsmandate zu garantieren, dann fallen diese als zwingende Folge von Überhangmandaten an.

13 Diese drei Mandate, die die CSU zu viel erhält, stellen weder klassische Überhangmandate noch Quasi-Überhangmandate dar, aber in gewisser Weise einen „Überhang an Mandaten“.

14 Vgl. zur Logik ausführlicher Joachim Behnke, Ein sparsames länderproporzoptimierendes parteienproporzgewährendes automatisches Mandatszuteilungsverfahren mit Ausgleich ohne negatives Stimmgewicht, in: ZParl, 43. Jg. (2012), H. 3, S. 675 - 693, S. 683 ff.; vgl. zum Verfahren selbst ders., Überhangmandate: Ein (behebbarer) Makel im institutionellen Design des Wahlsystems, in: ZPol, 13. Jg. (2003), H. 3, S. 1235 - 1269; Friedrich Pukelsheim, Bundeswahlgesetz - Nächste Etappe, in: DVBl, 123. Jg. (2008), H. 14, S. 889 - 897. 


\begin{tabular}{|l|r|r|r|r|r|r|}
\hline Tabelle 4: Endverteilung der Mandate nach neuem Wahlgesetz \\
\hline \multicolumn{1}{|c|}{ Bundesland } & SPD & CDU & CSU & Grüne & Linke & alle \\
\hline Baden-Württemberg & 20 & $43(44)$ & 0 & 10 & 5 & 78 \\
Bayern & 22 & 0 & 56 & 9 & 4 & 91 \\
Berlin & 8 & 9 & 0 & 4 & 6 & 27 \\
Brandenburg & 5 & $9(8)$ & 0 & 1 & 5 & 20 \\
Bremen & 2 & 2 & 0 & 1 & 1 & 6 \\
Hamburg & 5 & 5 & 0 & 2 & 1 & 13 \\
Hessen & 16 & 21 & 0 & 5 & 3 & 45 \\
Mecklenburg-Vorpommern & 3 & 6 & 0 & 1 & 3 & 13 \\
Niedersachsen & 25 & 31 & 0 & 6 & 4 & 66 \\
Nordrhein-Westfalen & 52 & $63(65)$ & 0 & 13 & 10 & 138 \\
Rheinland-Pfalz & 10 & 16 & 0 & 3 & 2 & 31 \\
Saarland & 3 & 4 & 0 & 1 & 1 & 9 \\
Sachsen & 6 & 17 & 0 & 2 & 8 & 33 \\
Sachsen-Anhalt & 4 & $9(8)$ & 0 & 1 & 5 & 19 \\
Schleswig-Holstein & 9 & 11 & 0 & 3 & 1 & 24 \\
Thüringen & 3 & $9(8)$ & 0 & 1 & 5 & 18 \\
\hline Bund & 193 & 255 & 56 & 63 & 64 & 631 \\
\hline Quelle: Eigene Berechnungen auf Basis der amtlichen Statistiken des Bundeswahlleiters. & \\
\hline \multicolumn{5}{|l|}{} \\
\hline
\end{tabular}

hangmandate erhält. Es werden dafür die letzten drei Listenmandate, die bei der Unterverteilung der 255 Mandate der CDU auf Landeslisten verteilt werden, in denen mehr Proporzmandate als Direktmandate anfallen, diesen Landeslisten abgezogen beziehungsweise erst gar nicht zugeteilt. Die drei Überhangmandate werden also durch den Wegfall von zwei Listenmandaten in Nordrhein-Westfalen und eines in Baden-Württemberg kompensiert. Allerdings erhalten die beiden Landeslisten aufgrund der Erhöhung der Gesamtsitzzahl in der Endverteilung nicht weniger Sitze, als ihnen nach der Grundverteilung zugestanden hätten (vgl. Tabelle 2). Es ist aber keineswegs sicher, dass alle Landeslisten nach dem Ausgleich besser gestellt sind als zuvor. Die bayerische Landesliste der SPD zum Beispiel erhält in der Endverteilung trotz der zehn zusätzlichen Ausgleichsmandate für die SPD auf der Bundesebene ein Mandat weniger als in der Grundverteilung. ${ }^{15}$ Ein solcher Mandatsverlust zwischen Grundverteilung und Endverteilung wirft wiederum weitere Probleme auf, auf die noch einzugehen sein wird.

15 Gelegentlich wird dieses Phänomen auch als „negativer Ausgleich“ bezeichnet. Dieser Ausdruckt ist allerdings irreführend, da der Ausgleich dort, wo er stattfindet, nämlich auf der Bundesebene, ja keineswegs negativ, sondern immer positiv ist. Die Verletzung der internen Monotoniebedingung (mehr Mandate für eine Partei insgesamt führen zu weniger Mandaten einer Landesliste) ist Folge der Inkonsistenz zwischen dem Verteilungskriterium der Bevölkerungszahlen auf der ersten Stufe und dem der zweiten Stufe, nämlich den Zweitstimmen. Dies hat zur Folge, dass die zweite Verteilung nicht zwangsläufig in die erste „eingebettet“ (Joachim Behnke, a.a.O. (Fn. 3), S. 6) ist, obwohl in der zweiten Verteilung insgesamt mehr Sitze verteilt werden. 


\section{Kritik des neuen Wahlgesetzes}

Zweifellos stellt das neue Wahlgesetz in einigen grundlegenden Aspekten eine deutliche Verbesserung gegenüber dem Wahlgesetz von 2008 dar. Dies betrifft vor allem die Geltung des Prinzips der Erfolgswertgleichheit, die durch den Ausgleich der Überhangmandate garantiert wird. ${ }^{16}$ Das demokratietheoretisch größte Problem des alten Wahlgesetzes von 2008 kann damit als gelöst betrachtet werden. ${ }^{17}$ Allerdings besitzt das neue einige schwerwiegende Mängel, so dass es sicherlich nicht als gelungen bezeichnet werden kann. Die negativen Seiten bestehen in dem Risiko einer starken Vergrößerung des Bundestags, einer unnötigen „Verfahrensrendite“ und einiger Absurditäten, die durch die Verwendung zweier unterschiedlicher Zuteilungskriterien auf der ersten und der zweiten Stufe entstehen.

\section{1. Überhangmandate und Erfolgswertgleichheit}

Der erfreulichste Aspekt des neuen Wahlgesetzes besteht darin, dass es das Problem der Überhangmandate gelöst hat. Diese stellten den mit Abstand größten Makel des alten Wahlrechts dar ${ }^{18}$, auch wenn sich - unglücklicherweise - die Wahlreformdebatte seit dem Urteil von 2008 vornehmlich auf den Effekt des negativen Stimmgewichts konzentriert hatte. Überhangmandate hatten aufgrund der Anzahl, mit der sie seit der Wiedervereinigung angefallen waren, das Potenzial, das politische Ergebnis einer Wahl entscheidend zu verändern, bis hin zu einer Umkehr der Mehrheitsverhältnisse. Die Legitimation einer Regierung, die sich auf Überhangmandate stützen würde, stünde aber auf wackeligen Füßen. ${ }^{19}$ Dieses Risiko ist nun gebannt, denn der Proporz wird zwischen den Parteien durch den Ausgleich - von Rundungsfehlern abgesehen - in nahezu perfekter Weise hergestellt. Damit ist die Sitzzahl jeder Partei durch ihre Zweitstimmen zu hundert Prozent gedeckt, und sowohl dem Erfordernis der Erfolgswertgleichheit bezüglich der Entscheidungen der Wähler als auch dem der Chancengleichheit zwischen den Parteien wird Genüge getan. Das neue Wahlgesetz beseitigt daher die grundlegenden Gerechtigkeitsprobleme, die dem alten inhärent waren.

\subsection{Negatives Stimmgewicht}

Maßgeblich war die Wahlrechtsdebatte zwischen dem Urteil des BVerfG von 2008 und dem von 2012 vor allem durch das Phänomen des so genannten negativen Stimmgewichts geprägt. ${ }^{20}$ Damit ist das Phänomen gemeint, dass eine Partei weniger Mandate erhalten

16 Vgl. Heiko Holste, Demokratie wieder flott gemacht: Das neue Sitzzuteilungsverfahren im Bundeswahlgesetz sichert das gleiche Wahlrecht, in: NVwZ, 32. Jg. (2013), H. 9, S. 529 - 534.

17 Eine ausführliche Darstellung findet sich hierzu in Hans Meyer, Die Zukunft des Bundestagswahlrechts. Zwischen Unverstand, obiter dicta, Interessenkalkül und Verfassungsverstoß, BadenBaden 2010.

$18 \mathrm{Vgl}$. hierzu insbesondere ebenda.

19 Vgl. ausführlicher Joachim Behnke, Grundsätzliches zur Wahlreformdebatte, in: APuZ, B4 I 2011, S. $14-21$, S. 19.

20 Erstmals beschrieben in Hans Meyer, Der Überhang und anderes Unterhaltsames aus Anlass der Bundestagswahl 1994, in: KritV, 77. Jg. (1994), S. 312 - 362. 
kann, wenn sie mehr Stimmen bekommt und umgekehrt mehr Mandate bei weniger Stimmen. Im neuen Wahlgesetz kann das negative Stimmgewicht nicht auf der Ebene der ersten Stufe auftreten, da die Sitzkontingente der Länder ja durch die Bevölkerungszahlen bestimmt sind. Veränderungen des Wahlergebnisses können daher nur zu Veränderungen der Verteilung der Sitze innerhalb eines Landes zwischen den Landeslisten der verschiedenen Parteien führen, aber eben nicht zu einer Veränderung der Verteilung der Sitze zwischen den Landeslisten einer Partei, da diese nicht miteinander verrechnet werden. Dies ist auch der Grund, warum die Landessitzkontingente aufgrund einer Größe bestimmt werden, die nicht durch die Wahlentscheidungen beeinflusst wird.

Allerdings kann es zum Auftreten des Effekts des negativen Stimmgewichts im Rahmen des Ausgleichs kommen. Um in Bezug auf ein bestimmtes Wahlergebnis das Ausmaß der „Anfälligkeit" für beziehungsweise die „Verwundbarkeit“ durch das negative Stimmgewicht $\mathrm{zu}$ bestimmen, lässt sich ein Index konstruieren, indem man ausgehend vom aktuellen Wahlergebnis systematisch Veränderungen der Stimmenzahlen vornimmt und beobachtet, in wie vielen Fällen diese Fluktuationen zu einem negativen Stimmgewicht führen. Dieser Index kann dann für den Vergleich verschiedener Wahlsysteme hinsichtlich ihrer Anfälligkeit für negatives Stimmgewicht herangezogen werden. Für ein bestimmtes Wahlergebnis ist es lediglich vonnöten, dass die simulierten Wahlergebnisse dieselben sind und in einem vernünftigen Bereich um das tatsächliche streuen, um den Vergleich zwischen zwei Wahlsystemen durchzuführen.

Um auch theoretisch zwischen verschiedenen Wahlergebnissen für dasselbe Wahlsystem vergleichen zu können, muss die Festlegung des Fluktuationsbereichs standardisiert erfolgen. Im Folgenden wurde für die Konstruktion eines solchen Index das Ausgangswahlergebnis systematisch so variiert, dass für jede Partei in jedem Bundesland die Zahl der Zweitstimmen um einen bestimmten Betrag in einem bestimmten Intervall verändert wurde. Das Intervall der Schwankungen der Stimmenzahlen wurde auf einen Bereich vom negativen Zweifachen der Hare-Quota ${ }^{21}$ bis zum positiven Zweifachen der Hare-Quota festgelegt. Die Schrittgröße zwischen zwei Simulationen beträgt 1.000 Stimmen. Für die Bundestagswahl 2013 beträgt die Hare-Quota zum Beispiel 61.651,2 Stimmen, also auf Tausender gerundet circa 62.000 Stimmen. Das Wahlergebnis jeder Partei wird daher in jedem Bundesland in 1.000er-Schritten so modifiziert, dass zwischen 0 und 124.000 Stimmen zu ihrem Zweitstimmenergebnis addiert beziehungsweise subtrahiert werden. Für jede Partei gibt es also in einem Bundesland 249 verschiedene Wahlergebnisse, die simuliert werden. Simulierte Stimmenergebnisse, bei denen die Stimmenzahl unter 0 fallen würde, gehen nicht in die Untersuchung ein. Insgesamt werden daher 15.164 verschiedene Wahlergebnisse simuliert. Von diesen tritt 2013 in 110 Fällen (vgl. Tabelle 5) ein negatives Stimmgewicht auf.

Die Inzidenz beträgt daher 110/15.164 = 0,0073, also 7,3 Promille. Das negative Stimmgewicht tritt 2013 unter den genannten Bedingungen des Simulationsmodells immer in Zusammenhang mit der SPD in Bayern auf. Zwischen 87.000 und 124.000 Stimmen weniger für die SPD in Bayern hätten zum Beispiel dazu geführt, dass sie insgesamt statt 193 Sitzen 194 Sitze erhalten hätte. Umgekehrt hätten zwischen 86.000 und 124.000

21 Die Hare-Quota entspricht der Stimmenzahl, die durchschnittlich auf einen zu vergebenden Sitz entfällt. Sie errechnet sich daher als Quotient aus der Gesamtstimmenzahl und der Gesamtzahl der zu vergebenden Sitze. 


\begin{tabular}{|c|c|c|c|c|}
\hline \multicolumn{6}{|c|}{ Tabelle 5: Instanzen des negativen Stimmgewichts nach neuem Wablgesetz } \\
\hline Land & Partei & Inkrement & Originalsitzzahl & Neue Sitzzahl \\
\hline Bayern & SPD & -124.000 bis -87.000 & 193 & 194 \\
Bayern & SPD & -86.000 bis -80.000 & 193 & 195 \\
Bayern & SPD & 60.000 bis 85.000 & 193 & 190 \\
Bayern & SPD & 86.000 bis 124.000 & 193 & 191 \\
\hline Quelle: Eigene Berechnungen.
\end{tabular}

Stimmen mehr dazu geführt, dass ihre Sitzzahl von 193 auf 191 gefallen wäre. Nach dem alten Wahlgesetz hätte sich ein negatives Stimmgewicht in insgesamt 554 der 15.164 Fälle ergeben, also eine Inzidenz von 0,0365, also 3,7 Prozent, mithin ungefähr fünfmal so hoch wie beim neuen. Allerdings kann man auch keineswegs behaupten, dass das negative Stimmgewicht beim neuen Wahlgesetz keine Rolle mehr spielt. Um den Effekt genauer zu analysieren, lohnt sich ein vertiefter Vergleich der beiden Wahlsysteme hinsichtlich der Konstellationen, in denen das negative Stimmgewicht auftritt.

\begin{tabular}{|r|l|c|r|c|c|}
\hline \multicolumn{6}{|c|}{ Tabelle 6: Instanzen des negativen Stimmgewichts nach altem Wahlgesetz von 2008} \\
\hline Nr. & \multicolumn{1}{|c|}{ Land } & Partei & Inkrement & $\begin{array}{c}\text { Original- } \\
\text { sitzzahl }\end{array}$ & $\begin{array}{c}\text { Neue } \\
\text { Sitzzahl }\end{array}$ \\
\hline 1 & Brandenburg & CDU & -124.000 bis -19.000 & 246 & 247 \\
2 & Brandenburg & CDU & 44.000 bis 60.000 & 246 & 245 \\
3 & Bremen & SPD & -117.000 bis -25.000 & 182 & 183 \\
4 & Hamburg & SPD & -124.000 bis -84.000 & 182 & 183 \\
5 & Hamburg & SPD & -83.000 bis -75.000 & 182 & 184 \\
6 & Hamburg & SPD & -74.000 bis -11.000 & 182 & 183 \\
7 & Mecklenburg-Vorpommern & CDU & -124.000 bis -29.000 & 246 & 247 \\
8 & Saarland & CDU & -124.000 bis -12.000 & 246 & 247 \\
9 & Saarland & CDU & 5.000 bis 60.000 & 246 & 245 \\
10 & Sachsen & CDU & -124.000 bis -98.000 & 246 & 247 \\
11 & Sachsen-Anhalt & CDU & -124.000 bis -22.000 & 246 & 247 \\
12 & Sachsen-Anhalt & CDU & 41.000 bis 60.000 & 246 & 245 \\
13 & Thüringen & CDU & -124.000 bis -14.000 & 246 & 247 \\
14 & Thüringen & CDU & 49.000 bis 60.000 & 246 & 245 \\
\hline Quelle: Eigene Berechnungen. & \multicolumn{4}{|}{} \\
\hline
\end{tabular}

Im alten Wahlgesetz tritt das negative Stimmgewicht hauptsächlich bei der CDU auf, gelegentlich aber auch bei der SPD. Nach dem alten Wahlgesetz wären ebenfalls vier Überhangmandate für die CDU angefallen und zwar in Brandenburg, Saarland, Sachsen-Anhalt und Thüringen. Die Phänotypen des negativen Stimmgewichts in den Zeilen 1, 2, 8, 9, 11, 12, 13 und 14 lassen sich daher genau auf die klassische Form des negativen Stimmgewichts im Zusammenhang mit Überhangmandaten zurückführen. In Mecklenburg-Vorpommern und in Sachsen erhielt die CDU gerade genauso viele Direktmandate, wie ihr nach Proporz dort zugestanden hätten. Das negative Stimmgewicht tritt daher hier nur in der einen Richtung auf, das heißt Stimmenverluste der CDU hätten dort die Entstehung eines Überhangmandats bewirkt und in der internen Verteilung woanders ein zusätzliches 
Listenmandat entstehen lassen. Ganz analog verhält es sich mit der SPD in Bremen, die dort zwei Direktmandate erzielen konnte, die ihr auch nach Proporz zugestanden hätten. In Hamburg führt ein Verlust der SPD von zwischen 83.000 und 75.000 Stimmen sogar dazu, dass sie insgesamt zwei Mandate mehr erhält. Dies liegt daran, dass die SPD in der Oberverteilung trotz des Stimmenverlusts kein Mandat weniger erhält und gleichzeitig wegen der fehlenden Stimmen in Hamburg zwei Überhangmandate entstehen. Dies stellt jedoch eine Ausnahme dar. Im alten Wahlsystem von 2008 entstehen negative Stimmgewichte typischerweise an den Rändern von Rundungseffekten innerhalb der Unterverteilung, ohne dass sich etwas an der Oberverteilung der Sitze ändert. Der Effekt kann aber überall auftreten, wo Überhangmandate vorhanden sind oder das Ergebnis knapp am Entstehen von Überhangmandaten vorbeigegangen ist.

Da im neuen Wahlgesetz der Effekt des negativen Stimmgewichts über den Ausgleich stattfindet, ergibt er sich in den Bundesländern, in denen die Partei, an der sich der Ausgleich orientiert, besonders stark überrepräsentiert ist. Im Falle der Wahl von 2013 ist dies Bayern. Gewinnt die SPD dort zwischen 60.000 und 85.000 mehr Stimmen, erhält die CSU bei der bayerninternen Verteilung der 92 Sitze einen Sitz weniger, also nur noch 55. Dies hat zur Folge, dass die CSU weniger überrepräsentiert ist; für den Ausgleich muss die Sitzzahl nur noch auf 621 erhöht werden. Die SPD bekommt dann nur noch sechs Ausgleichsmandate statt zehn. Da sie in der Grundverteilung ein Mandat mehr erhalten hat, bleibt es allerdings bei insgesamt drei Sitzen weniger. Hätte die SPD 240.000 Stimmen mehr in Bayern gewonnen, dann wären ihr sogar fünf Mandate insgesamt weniger zugefallen. Im neuen Wahlgesetz tritt das negative Stimmgewicht also auch in den höheren Bereichen der Veränderungen der Stimmenzahlen immer noch auf. Der Unterschied der Auftretenswahrscheinlichkeit zwischen altem und neuem Gesetz wird daher umso geringer, je größer die Bandbreite der Veränderungen wird, die man untersucht.

Allerdings kommt das negative Stimmgewicht, wenn man vom konkreten Ergebnis von 2013 ausgeht, selten vor, weil sich der Ausgleich an der CSU orientiert. Das Entstehen eines negativen Stimmgewichts kann also nur durch Stimmenveränderungen in Bayern erfolgen. Richtet sich der Ausgleich jedoch an einer anderen Partei aus, kann das negative Stimmgewicht in deutlich mehr Bundesländern auftreten.

Ändert man das Ergebnis von 2013 etwa leicht ab, indem man die Zweitstimmen aller bayerischen Landeslisten um acht Prozent erhöht und verringert die Anzahl der Direktmandate der CDU leicht, so dass sie keine Überhangmandate erhält, dann orientiert sich der Ausgleich an den Grünen. Führt man wieder die Simulationen zur Konstruktion des Indikators der Verwundbarkeit durch ${ }^{22}$, so beträgt die Inzidenzrate eines negativen Stimmgewichts jetzt immerhin 2,5 Prozent. Der Phänotyp entspricht dabei dem folgenden Muster: Stimmverluste einer Partei in einem Bundesland führen dort zu einem zusätzlichen Mandat für die Grünen, dadurch erhöht sich der Grad, in dem diese überrepräsentiert sind, es erfolgt ein größerer Ausgleich mit Sitzgewinnen auch für die Partei, die ursprünglich Stimmen verloren hat.

Nimmt man dieselbe Ausgangslage (Erhöhung der bayerischen Landeslisten), belässt aber der CDU ihre Überhangmandate, orientiert sich der Ausgleich zunächst an der CDU. Stimmenveränderungen können aber dann zu einer Erhöhung der Mandatszahl der Grü-

22 Um den Rechenaufwand herunterzusetzen mit den Intervallgrenzen -120.000 und 120.000 und Schrittgrößen von 10.000 . 
nen führen, wodurch sich der Ausgleich jetzt an den Grünen orientiert und auf diese Weise wieder den Effekt des negativen Stimmgewichts mit einer Inzidenz von 2,0 Prozent herbeiführt. Eine noch radikalere Simulation, bei der die Zweitstimmen in den neuen Ländern jeweils um 15 Prozent reduziert wurden, führt zu einer erheblichen Vergrößerung des Bundestags, wobei sich der Ausgleich nun an den Linken ausrichtet. In diesem Modell kommt es zu einer Inzidenzrate des negativen Stimmgewichts von 9,2 Prozent.

Das negative Stimmgewicht ist also mit dem neuen Wahlsystem keineswegs ein für alle Mal verbannt worden, sondern tritt über den Ausgleich in annähernd derselben Größenordnung wie beim alten Wahlsystem auf. Allerdings handelt es sich hierbei „nur“ um die Form eines so genannten „absoluten negativen Stimmgewichts“23. Von einem solchen spricht man, wenn mehr Stimmen für eine Partei zu weniger Sitzen führen oder umgekehrt, unabhängig von Änderungen bei der Sitzverteilung der übrigen Parteien. Bleiben die Sitzverteilungen der anderen Parteien hingegen konstant, ist ein „absolutes negatives Stimmgewicht“ zugleich ein „relatives negatives Stimmgewicht“, da dann mehr Stimmen nicht nur weniger Sitze bedeuten (und umgekehrt), sondern auch eine Verminderung des Sitzanteils. ${ }^{24}$ Da es beim alten Wahlgesetz keinen Ausgleich der Überhangmandate gab, trat das negative Stimmgewicht als absolutes wie auch als relatives auf. Dies stellt aus normativer Sicht eindeutig ein wesentlich größeres Problem dar, da es für den Wähler vermutlich nicht so sehr darauf ankommt, ob seine Stimme zu einem zusätzlichen Sitz für seine Partei führt, sondern darauf, ob sie ihr einen Vorteil im Wettstreit mit den anderen Parteien verschafft. Insofern könnte man das Problem des negativen Stimmgewichts als durch das neue Wahlsystem gelöst betrachten, da es das demokratietheoretisch bedenkliche relative negative Stimmgewicht verhindert (abgesehen von Rundungseffekten) und nur noch die deutlich harmlosere Form des absoluten negativen Stimmgewichts zulässt. Dann wäre allerdings auch das Ausgleichsmodell der SPD von 2011 ebenfalls in der Lage gewesen, das Problem des negativen Stimmgewichts zu beseitigen. Das wesentliche Argument, mit dem dieses in der Debatte aber seinerzeit von CDU/CSU und FDP abgelehnt worden war, beruhte darauf, dass damit nur das relative negative Stimmgewicht verhindert werde, nicht aber das absolute, das das verfassungsrechtlich bedenkliche sei. ${ }^{25}$ Nimmt man dieses Argument ernst, verstößt das neue Wahlgesetz in diesem Sinne genauso gegen das Gebot, ein negatives Stimmgewicht zu vermeiden wie das alte. Der Unterschied zwischen altem und neuem Gesetz besteht dann nur darin, an welcher Stelle das absolute negative Stimmgewicht auftritt. Während dies beim SPD-Entwurf vor dem Ausgleich geschieht, wird es beim neuen

23 Auch Martin Fehndrich kommt in seiner Stellungnahme zu dem interfraktionellen Gesetzentwurf (vgl. A-Drs. 17(4)634-C) zu diesem Ergebnis, wobei er die Effekte als „NSTG-ähnliche Effekte“ bezeichnet, da es sich seiner Ansicht nach um ein „echtes“ absolutes negatives Stimmgewicht nur dann handelt, wenn sich die Sitzzahlen der anderen Parteien nicht verändern. Dies ist allerdings eine Einschränkung, die nirgends aus den diesbezüglichen Aussagen des Bundesverfassungsgerichts abzuleiten ist. Auch Ulrich Wiesner bezeichnet diese Effekte in Übereinstimmung mit der hier vertretenen Ansicht ebenfalls als „absolutes negatives Stimmgewicht“ (vgl. ders., Sitzzuteilungsverfahren, Stand: 25. Oktober 2013, http://ulrichwiesner.de/stimmgewicht/, Abruf am 14. Dezember 2013).

24 Vgl. hierzu auch Daniel Lübbert, Zur Berechnung negativer Stimmgewichte. Methodik und Ergebnisse der Prüfung aktueller Wahlrechtsmodelle, Info-Brief der Wissenschaftlichen Dienste des Deutschen Bundestages, WD 8 - 3000 - 127/2011.

25 Vgl. Bernd Grzeszick, Stellungnahme zu den Gesetzentwürfen zur Änderung des Bundeswahlgesetzes, Ausschussdrucksache 17(4) 327D 2011. 
Gesetz erst durch den Ausgleich induziert. Der Zeitpunkt des Auftretens aber dürfte unerheblich für die Beurteilung sein. Entweder ist also das neue Wahlgesetz verfassungswidrig, oder ein Ausgleich im Sinne des SPD-Modells wäre es nie gewesen. Dies ist insofern von großer Bedeutung, als sich hierdurch der große Umweg über die Bevölkerungszahlen als erstes Zuteilungskriterium als überflüssig erweist, wenn das negative Stimmgewicht als unproblematisch angesehen wird, sobald ein Ausgleich stattfindet. ${ }^{26}$ Dieser Umweg, das heißt die Verwendung zweier unterschiedlicher Zuteilungskriterien im Verlauf des Verfahrens führt aber zur größten Inkonsistenz und Absurdität des neuen Verfahrens in Form der so genannten Verfahrensrendite.

\subsection{Die Verfahrensrendite}

Es waren nicht wenige, denen das letzte Wahlergebnis ein Rätsel aufgab, das darin bestand, wie es zu insgesamt 33 zusätzlichen Mandaten kommen konnte, obwohl die CDU nur vier Überhangmandate erzielt hatte. Wer sich schon darüber wundern konnte, wäre sicherlich noch irritierter gewesen, wenn er erfahren hätte, dass es zu diesen 33 zusätzlichen Mandaten genauso gekommen wäre, wenn die CDU kein einziges Überhangmandat erhalten hätte, denn diese zusätzlichen Mandate kommen zustande, um die Überrepräsentation der CSU auf der ersten Stufe auszugleichen. Diese Überrepräsentation ist aber vollkommen unabhängig von Überhangmandaten, denn die CSU hatte aufgrund ihres sehr guten Ergebnisses ja Anspruch auf mehr Proporzmandate, als sie Direktmandate gewonnen hatte. Sie verdankt sich - wie schon gezeigt - allein dem Umstand, dass man auf der ersten Stufe die Bevölkerungszahlen für die Zuteilung der Landessitzkontingente heranzieht. Würden die Sitze durchgehend auf jeder Stufe auf der Basis der Zweitstimmen vergeben, wäre diese Überrepräsentation gar nicht erst entstanden. Der dadurch notwendig gewordene Ausgleich ist daher überflüssig. Dieser „überflüssige Ausgleich“ kann daher auch als „Verfahrensrendite“27 bezeichnet werden. Er kann in keiner Weise mit inhaltlichen Argumenten begründet werden, ist einzig und allein die Folge der Konstruktion des Wahlsystems. Natürlich ist dies in gewisser Weise eine Tautologie, denn jeder Effekt, den ein Wahlsystem hervorbringt, ist Folge seiner Gestaltung. Hier jedoch ist die nähere Bestimmung „einzig und allein“"wörtlich zu nehmen, denn die Designelemente, die den überflüssigen Ausgleich schaffen, verfolgen keinen Zweck mehr beziehungsweise finden jenseits des durch das Design ursprünglich verfolgten Zwecks statt. Die ursprünglich mit dem Ausgleich verfolgte Intention des Gesetzgebers war offensichtlich, die durch Überhangmandate entstandenen Proporzverzerrungen zu neutralisieren. Doch durch die unglückliche Berechnung der Landessitzkontingente anhand der Bevölkerung kommt es schon hier zu oft nicht unerheblichen Proporzverzerrungen, die - wie auch im Fall der Bundestagswahl 2013 - sogar die durch Überhangmandate bewirkten deutlich übersteigen können. Die ganze Fragwürdigkeit eines solchen zweckfreien designbedingten Ausgleichs wird klar, wenn man sich die

26 Zur Beschreibung eines Ausgleichsmodells in Form des SPD-Gesetzesentwurfs vgl. Daniel Lübbert / Felix Arndt / Friedrich Pukelsheim, Proporzwahrende Anpassung der Bundestagsgröße - ein Lösungsvorschlag für das Problem der negativen Stimmgewichte bei Bundestagswahlen, in: ZParl, 42. Jg. (2011), H. 2, S. $426-435$.

27 Joachim Behnke, a.a.O. (Fn. 3), S. 7. 
Ursachen, die zum Ausgleich führen, klar macht: Es sind im Wesentlichen zwei Faktoren, die zur Überrepräsentation des bayerischen Sitzkontingents führen beziehungsweise zum unterdurchschnittlichen Anteil der zu verrechnenden Zweitstimmen an der Bevölkerung. Der erste Faktor liegt in der um ungefähr zwei Prozent niedrigeren Wahlbeteiligung als im Bundesdurchschnitt. Der zweite Faktor ist darin begründet, dass in Bayern von den abgegebenen Zweitstimmen fast drei Prozent weniger auf die Parteien entfielen, die auch im Bundestag vertreten sind, als im Bundesdurchschnitt. ${ }^{28}$

Ein weiteres Problem der Verfahrensrendite besteht darin, dass sie - wie gezeigt - einen günstigen Nährboden für die Entstehung des Effekts des negativen Stimmgewichts darstellt. Eine durch die Verfahrensrendite bewirkte Verzerrung wirkt sich stärker zur Ausbildung von negativen Stimmgewichtseffekten aus als es der Fall gewesen wäre, wäre sie durch Überhangmandate zustande gekommen. Außerdem gilt: je größer die Verfahrensrendite, desto größer die Anfälligkeit für den Effekt des negativen Stimmgewichts. Für Ironiker (oder Zyniker) des Prozesses politischer Reformen muss dies eine Freude sein: Um das negative Stimmgewicht zu beseitigen, entschließt sich die Union zur Trennung der Landeslisten, berechnet aber im ersten Anlauf diese Sitzkontingente anhand der Wählerzahlen, was als verfassungswidrig erkannt wird. Um diesem Problem zu entgehen, werden die Sitzkontingente anhand der Bevölkerungszahlen berechnet, wodurch tatsächlich der negative Stimmgewichtseffekt an dieser Stelle nicht mehr auftreten kann. Die durch die Verwendung des Maßstabs der Bevölkerungszahlen entstandenen Verzerrungen aber sollen durch einen Ausgleich neutralisiert werden, durch den das Verfahren mit dem Virus des negativen Stimmgewichtseffekts erneut infiziert wird (wenn auch nur mit einer schwächeren Mutation desselben).

\subsection{Mandatsverlust zwischen Grundverteilung und Endverteilung}

Die unglückliche Verwendung zweier unterschiedlicher Maßstäbe bei der Sitzverteilung in der ersten und zweiten Stufe hat neben der überflüssigen Verfahrensrendite noch einen weiteren problematischen Effekt. Es kann nämlich passieren, dass bestimmte Landeslisten trotz des Ausgleichs am Ende weniger Sitze erhalten, als sie in der Grundverteilung zugewiesen bekämen. Dies ist bei der Wahl von 2013 zum Beispiel für die bayerische Landesliste der SPD der Fall. Je kleiner der Ausgleich ausfällt, desto wahrscheinlicher ist dieser Effekt, das heißt desto mehr Landeslisten werden davon betroffen sein. Bei der oben durchgeführten Simulation, die sich an der CDU orientierte (also unter Neutralisierung der Überrepräsentation des bayerischen Kontingents), wäre es zum Beispiel in fünf Fällen zu einer solchen Inkonsistenz gekommen, an denen drei Parteien beteiligt gewesen wären.

Eine dieser Parteien wäre die CDU gewesen, die nach dem Ausgleich in Baden-Württemberg zwei Mandate weniger und in Hessen ein Mandat weniger erhalten hätte als in der Grundverteilung. Dies ist offensichtlich absurd. Der Ausgleich würde so durchgeführt, dass die Sitzzahl des gesamten Parlaments so lange erhöht wird, bis der Gesamtsitzanspruch der

28 Genauer zu den Ursachen der Überrepräsentation vgl. Joachim Behnke, a.a.O. (Fn. 6). Kritisch hierzu vgl. auch Florian Grotz, a.a.O. (Fn. 3); Eckhard Jesse, Nach allen Seiten offen? Der Ausgang der Bundestagswahl 2013 und mögliche Folgen für das Parteiensystem und das Koalitionsgefüge, in: Zeitschrift für Politik, 60. Jg. (2013), H. 4, S. 374 - 392, S. 386. 
CDU aus der Grundverteilung abgegolten wäre. Dieser Gesamtsitzanspruch setzt sich jedoch zusammen aus den Sitzansprüchen der CDU in den einzelnen Ländern. Nach dem Ausgleich aber hätten einige Landeslisten eine geringere Anzahl an Sitzen als die aus der sich der Gesamtsitzanspruch ihrer Partei überhaupt erst gebildet hätte, der wiederum konstitutiv für die Durchführung des Ausgleichs ist. Im Laufe der Durchführung des Ausgleichs entzieht sich das Verfahren gewissermaßen die Grundlage, die Anlass ihrer Durchführung war. Das ist so, als ob man sich beim Aufstehen selbst gleichzeitig die Beine wegziehen würde oder - für Liebhaber von Science-Fiction und Zeitreisenparadoxien - so, als ob ein Zeitreisender in der Vergangenheit die Umstände verändert, die erst zu seiner Geburt geführt haben.

Dieses Problem war den Verfassern des Gesetzes sehr wohl bewusst; dies ist auch der Grund, warum der Begriff des „Sitzanspruchs“ tunlichst vermieden wird. ${ }^{29}$ Vielmehr wird die Interpretation gepflegt, es handle sich bei der ersten Stufe nur um eine Art von vorgeschalteter „Strohmannverteilung “30, „Überschlagsrechnung“31 oder „Vorabkalkulation“32 zur Festlegung der Parlamentsgröße, so dass auf der Basis dieser Zahl dann auf der zweiten Stufe die „eigentliche“ Sitzverteilung entsprechend der Zweitstimmen beginnen kann. In seinem Urteil von 2008 hat das Bundesverfassungsgericht jedoch auch festgestellt: „Ein Wahlsystem, auf dem die Mandatsverteilung beruht, muss grundsätzlich frei von willkürlichen und widersinnigen Effekten sein. "33 Demnach kann die Festlegung einer so bedeutenden Größe wie die Zahl der Parlamentssitze ebenfalls nicht willkürlich sein, es muss eine stichhaltige und nachvollziehbare Begründung geben, warum von der im Gesetz vorgegebenen Normzahl von 598 abgewichen wird. ${ }^{34}$ Es kann dabei „nicht darum gehen, ein Rechenverfahren zu finden, mit dem man ,irgendwie' die Größe des Parlaments festlegt, sondern die Anwendung dieses spezifischen Rechenverfahrens muss so begründet werden, dass die arithmetischen Operationen als mathematische Übersetzung eines normativ begründeten Verteilungsprinzips interpretiert werden können“"35. Ginge es nur um die Herstellung der Proportionalität, wäre jede halbwegs große Sitzzahl geeignet, dies problemlos zu gewährleisten. Doch die Intention des Gesetzgebers liegt offensichtlich darin, die Sitzzahl nur im notwendigen Maß zu erhöhen, also von der vorgegebenen Normzahl so wenig wie mög-

29 Allerdings spricht auch das neue Gesetz in $\$ 6$ Abs. 2 von einer „ersten Verteilung“, nicht von einer „vorläufigen Verteilung“ oder einer „Quasi-Verteilung“. Wie es nun möglich sein soll, dass etwas „verteilt“ werden kann, ohne dass sich daraus ein Anspruch ableiten lässt, ist unklar und widerspricht dem alltagsüblichen Gebrauch des Begriffs „Verteilen“.

30 Joachim Behnke, a.a.O. (Fn. 3), S. 6.

31 Vgl. Martin Fehndrich / Wilko Zicht / Matthias Cantow, Wahlsystem der Bundestagswahl, letzte Aktualisierung am 2. Oktober 2013, http://www.wahlrecht.de/bundestag/ (Abruf am 14. Dezember 2013).

32 Friedrich Pukelsheim / Matthias Rossi, a.a.O. (Fn. 3).

33 BVerfG, 2 BvC 1/07 vom 3. Juli 2008, Absatz-Nr. (1 - 145), Abs. 105.

34 Durch Rundungseffekte bei der Vergabe der Sitze in den Ländern, gibt es so gut wie immer eine Partei, bei der diese Rundungseffekte, aufsummiert über alle 16 Länder, zu einer Überrepräsentation führen müssen. Da sich der Ausgleich an der am stärksten überrepräsentierten Partei ausrichtet, heißt dies, dass es allein aufgrund der Rundungseffekte zu einer Vergrößerung des Bundestags kommt und die Normgröße in der Regel verfehlt wird, vgl. hierzu auch Philipp Weinmann, Führt das Wahlrecht zur „Aufblähung“ des Bundestages? Simulationsrechnungen auf Basis des neuen Bundeswahlgesetzes, in: ZParl, 44. Jg. (2013), H. 4, S. 719 - 741.

35 Joachim Behnke, a.a.O. (Fn. 9). 
lich abzuweichen. Die „Notwendigkeit“ besteht aber augenscheinlich darin, dass jede Partei nach dem Ausgleich mindestens so viele Sitze erhält, wie ihr im Sinne eines dahinterstehenden Fairness- und Gerechtigkeitsgebots zustehen. Es ist daher unwesentlich, ob man das, was einer Partei gerechterweise zusteht, nun mit dem Begriff des Sitzanspruchs belegt oder nicht - in der Sache kann es sich nur um einen solchen handeln, wenn damit eine Vergrößerung des Parlaments gerechtfertigt werden soll. Wenn nun aber der Gesamtsitzanspruch, den sich eine Partei in der Grundverteilung erwirbt und mit dem sie in die Phase des Ausgleichs eintritt, genau als solcher Anspruch behandelt werden soll, dann muss dies auch für die Komponenten gelten, aus denen er sich erst zusammensetzt. Es ist ja ein Grundzug jedes Ausgleichsverfahrens, dass es die Bedingung erfüllt, dass die Situation nach dem Ausgleich gegenüber der vorherigen pareto-superior sein muss, wie Ökonomen sagen würden, das heißt die Besserstellung einzelner darf nicht durch eine Verschlechterung anderer erkauft werden, selbst dann nicht, wenn die Anzahl derjenigen, die sich besser stellen, die Anzahl derjenigen, die sich schlechter stellen, übertrifft. ${ }^{36}$

Natürlich ist es allerdings richtig, dass man den Landeslisten, die in der Endverteilung gegenüber der Grundverteilung verlieren, nur etwas wegnimmt, worauf sie niemals einen moralisch begründeten Anspruch hatten, denn der Sitzanspruch kann sich letztlich immer nur auf Wählerstimmen und nicht auf Bevölkerungszahlen gründen. Aber dann ist eben die Heranziehung dieser moralisch nicht zu rechtfertigenden Sitzzahlen zur Konstruktion des Gesamtsitzanspruchs als Grundlage für den Ausgleich offenkundig willkürlich.

Wie schon gezeigt, sind die auf der Bevölkerungszahl basierenden Sitzzahlen irreführend. Teilweise entstehen hier Quasi-Überhangmandate in der Grundverteilung, die es niemals gegeben hätte, wenn man sich von vornherein an den Zweitstimmen orientiert hätte, die ja der einzige Maßstab für Sitzverteilungen sind, der (moralisch und demnach auch im Sinne des Gesetzes ${ }^{37}$ ) gerechtfertigt werden kann. Umgekehrt aber kann es auch zu „camouflierten Überhangmandaten “38 kommen. Dies tritt dann ein, wenn es in bestimmten Ländern auf der ersten Stufe zu einer Überflutung von Mandaten kommt, die durch die Zweitstimmen bei weitem nicht gedeckt sind. Dies wäre zum Beispiel in besonders dramatischer Weise in den neuen Ländern der Fall, wenn die Linke, wie zum Beispiel 2002 schon geschehen, nicht in den Bundestag einziehen würde. Diese Überrepräsentation der neuen Länder muss aber keineswegs zu einem Ausgleich in größerer Zahl führen. Es kommt dann auf der zweiten Stufe letztlich zur Anwendung des alten Wahlsystems von 2008, bereichert

36 Es ist genau eine Paradoxie dieser Art, nämlich das so genannte Alabama-Paradox (vgl. Michel Balinski / H. Peyton Young, Fair Representation. Meeting the Ideal of One Man, One Vote. New Haven / London 1982), das zu der verbreiteten Umstellung von Hare-Niemeyer zu Sainte-Laguë geführt hat, obwohl beide in Hinsicht auf Proportionalität gleich gut abschneiden. Das AlabamaParadox besagt, dass eine Partei schlechter abschneiden kann, wenn die Anzahl der zu verteilenden Sitze erhöht wird. Wenn ein Effekt aber - der bei der Verteilung von Sitzen zwischen Parteien auftritt - als widersinnig eingestuft wird, dann muss das auch für die Verteilung von Sitzen innerhalb einer Partei auf ihre Landeslisten zutreffen.

37 Aber das Gesetz ist eben so formuliert und muss so formuliert sein, dass es moralischen Ansprüchen gerecht wird. Das Gesetz folgt der Moral beziehungsweise dem, was wir für moralisch geboten halten. Allerdings sehen dies - zugegeben - Anhänger einer positivistischen Rechtstheorie in der Tradition John Austins anders, und offensichtlich gibt es gerade im Zusammenhang mit Wahlrechts- und den damit verbundenen Legitimationsfragen gar nicht so wenige Anhänger dieser Sichtweise.

38 Joachim Behnke, a.a.O. (Fn. 3), S. 7. 
um die Komponente des Ausgleichs. Dann ist es durchaus möglich, dass auf der zweiten Stufe in den neuen Ländern Überhangmandate vorhanden sind, die zuvor wegen des zu hohen Pegelstands an Mandaten nicht sichtbar geworden sind. Diese Überhangmandate werden mit Listenmandaten der betroffenen Partei in anderen Bundesländern verrechnet. Faktisch kommt es also zur Anwendung des Wahlsystems, wie es im Entwurf der Grünen von 2011 vorgesehen war. Genau diese Kompensation von Überhangmandaten durch Listenmandate, die vor allem auf Kosten der Landeslisten großer Bundesländer wie Nordrhein-Westfalen, Baden-Württemberg, Bayern und Niedersachsen gehen würde (soweit in diesen zur Kompensation heranzuziehende Listenmandate vorhanden sind), war aber von den großen Parteien und vor allem von der CDU vehement abgelehnt worden mit dem Argument, dass dadurch die Disproportionalität zwischen den Ländern, wie sie durch die Überhangmandate schon geschaffen ist, noch einmal verstärkt würde.

\subsection{Die potenzielle Vergrößerung des Bundestags}

Die im Vorfeld der Bundestagswahl am stärksten in der Öffentlichkeit diskutierte Schwäche des neuen Wahlgesetzes war die unter bestimmten Umständen mögliche enorme Vergrößerung des Bundestags. Das Ergebnis von 2009 zum Beispiel hätte zu einer Vergrößerung um insgesamt 73 Sitze auf 671 Sitze geführt. ${ }^{39}$ Der Ausgleich hätte sich bei diesem Ergebnis an der CDU orientiert, die durch ihre 22 Überhangmandate auf der ersten Stufe stark überrepräsentiert gewesen wäre. Diese Überhangmandate wären durch 51 weitere Sitze an die anderen Parteien aufgewogen worden, wobei einige dieser Ausgleichssitze in Überhangmandate von CSU und SPD in der Grundverteilung aufgegangen wären. Grob kann man also sagen, dass die 22 Mandate, die die CDU im Verhältnis zu ihren Zweitstimmen „zu viel“ erhalten hätte, durch etwas mehr als das Doppelte an Ausgleichsmandaten kompensiert worden wären. Dieses Verhältnis von benötigten Ausgleichsmandaten zur Anzahl der überzähligen Mandate der am stärksten überrepräsentierten Partei kann man als Hebeleffekt bezeichnen. Je kleiner die Partei, an der sich der Ausgleich orientiert, desto stärker der Hebeleffekt. Das größte Potenzial für den Hebeleffekt besitzt die CSU - zum einen schon deshalb, weil der Hebeleffekt lediglich wegen ihrer Größe einen bedeutenden Umfang hätte, und zweitens kommt noch erschwerend hinzu, dass die Überrepräsentation der CSU in Bayern immer gleich ihrer bundesweiten Überrepräsentation entspricht, da der Effekt nicht durch die entsprechende normale Repräsentation oder sogar Unterrepräsentation in anderen Bundesländern gedämpft wird. Die CDU hätte nach dem neuen Wahlgesetz zum Beispiel in der Grundverteilung 37 Mandate in Baden-Württemberg erhalten, obwohl ihr nach Proporz nur 28 zugestanden hätten, sie war dort also um ungefähr 32 Prozent überrepräsentiert. Bundesweit aber war sie nur um 13 Prozent überrepräsentiert. Je nachdem, wie das Stimmenergebnis der CSU ausfällt, kann der Hebeleffekt daher bis zur Größenordnung von 20 und mehr ansteigen.

Um das Potenzial von Überhangmandaten der CSU für die Vergrößerung des Bundestags aufzuzeigen, wurde wieder auf eine Simulation zurückgegriffen. Ausgehend von ihrem Ergebnis bei der Bundestagswahl 2013 von circa 3,2 Millionen Zweitstimmen wird die 
Zweitstimmenzahl der CSU in Schritten von 100.000 reduziert, beginnend bei 400.000 bis zu einer um eine Million reduzierten Stimmenzahl. Die der CSU entgehenden Stimmen werden zur Hälfte auf die SPD und zur Hälfte auf die Grünen verteilt. ${ }^{40}$ Darüber hinaus wird davon ausgegangen, dass die CSU weiterhin immer alle 45 Direktmandate gewinnt. Die entsprechenden Ergebnisse sind in Tabelle 7 zu sehen. Das erste Überhangmandat der CSU wäre entstanden, wenn sie 700.000 Zweitstimmen weniger erhalten hätte, was einem Zweitstimmenanteil von circa 38 Prozent entspricht, gegenüber den circa 49 Prozent, die sie bei der letzten Wahl tatsächlich erhalten hat. Der Bundestag hätte sich dann auf 646 Sitze vergrößert.

\begin{tabular}{|c|c|c|c|c|}
\hline \multicolumn{6}{|c|}{ Tabelle 7: Vergrößerung des Bundestags aufgrund von Überhangmandaten der CSU } \\
\hline Veränderung & $\begin{array}{c}\text { Anteil Zweit- } \\
\text { stimmen CSU }\end{array}$ & $\begin{array}{c}\text { Überhangmandate } \\
\text { CSU }\end{array}$ & $\begin{array}{c}\text { Bundestags- } \\
\text { größe }\end{array}$ & $\begin{array}{c}\text { Vergrößerung } \\
\text { Bundestag }\end{array}$ \\
\hline-400.000 & 42,9 & 0 & 629 & 31 \\
-500.000 & 41,4 & 0 & 626 & 28 \\
-600.000 & 39,9 & 0 & 620 & 22 \\
-700.000 & 38,3 & 1 & 646 & 48 \\
-800.000 & 36,8 & 3 & 672 & 74 \\
-900.000 & 35,3 & 4 & 700 & 102 \\
-1.000 .000 & 33,8 & 6 & 731 & 133 \\
\hline Quelle: Eigene Berechnungen.
\end{tabular}

Erhöht man den Verlust der CSU weiter sukzessive, erhält sie bei einem Zweitstimmenanteil von circa 34 Prozent sechs Überhangmandate, und der Bundestag müsste auf insgesamt 731 Sitze vergrößert werden. Bei der Simulation wird davon ausgegangen, dass alle anderen Ergebnisse konstant bleiben. Die betrachteten Effekte sind daher bedingt für die Ausgangssituation der Bundestagswahl 2013 mit den entsprechenden Modifikationen. Bei einem anderen Wahlergebnis wäre es durchaus möglich, dass die CSU auch schon fünf bis sechs Überhangmandate erhalten könnte, wenn sie nur knapp unter einem Zweitstimmenanteil von 40 Prozent landen würde. Der Hebeleffekt läge dennoch vermutlich zwischen 15 und 20. Betrachtet man den langfristig wirkenden strukturell bedingten Prozess der Erosion der Volksparteien, dann ist es nur eine Frage der Zeit, wann sich auch die CSU auf diesem Niveau befinden wird. Zwar ist es ihr wie bei der aktuellen Wahl bei entsprechend günstigen Umständen und einem sehr populären Kandidaten immer noch möglich, sehr gut abzuschneiden (wobei dieses Ergebnis - mit den früheren Traumergebnissen der CSU verglichen - ja ebenfalls nicht über die Tendenz nach unten hinwegtäuschen kann); die geringere parteipolitische Prägekraft der sozialstrukturellen Milieus und die daraus folgende größere Wählervolatilität ${ }^{41}$ führen aber zwangsläufig dazu, dass auch die ehemals großen Volkspar-

40 Für den untersuchten Effekt ist die konkrete Verteilung unwichtig, da am Schluss die Vergrößerung des Bundestags nur vom Zweitstimmenanteil der CSU abhängen wird, das heißt, der Bundestag wird solange vergrößert, bis mit diesen Zweitstimmen ihre errungenen Direktmandate abgegolten werden können.

41 Vgl. Kai Arzheimer, Mikrodeterminanten des Wahlverhaltens: Parteiidentifikation, in: Oscar W. Gabriel / Bettina Westle (Hrsg.), Wählerverhalten in der Demokratie. Eine Einführung, BadenBaden 2012, S. 223 - 246. 
teien, für die absolute Mehrheiten einmal eine Selbstverständlichkeit waren, zumindest in den Wahlen, die für sie unter ungünstigen Umständen stattfinden, sich in der 30-ProzentZone wiederfinden können. Die Geschichte der SPD in Nordrhein-Westfalen oder die der CDU in Baden-Württemberg sind Beleg hierfür.

Die neue Struktur des Parteiensystems, kleinere große Parteien und im Durchschnitt größere kleine Parteien als noch zwei Jahrzehnte zuvor würden unter dem neuen Wahlsystem allerdings auch jenseits des besonders dramatischen Hebeleffekts der CSU zu erheblichen Vergrößerungen des Bundestags führen. Detaillierte Simulationsergebnisse von Philipp Weinmann zeigen, dass auch Stimmenverteilungen, wie sie zwischen Sommer 2010 und Sommer 2011 in den Umfragen zur Sonntagsfrage erhoben wurden, zu sehr dramatischen Größeneffekten geführt hätten. ${ }^{42}$ Von diesen Ergebnissen kann mit guten Gründen angenommen werden, dass sie im Bereich dessen liegen, was unter realistischen Umständen möglich ist. Die Simulationen der Studie von Weinmann verdeutlichen sehr klar, dass die Vergrößerung 2013 nur deshalb so gering ausfiel, weil die CDU im Vergleich zu den vorhergehenden Wahlen so gut abschnitt. Situationen hingegen, in denen sowohl CDU als auch SPD deutlich unter 40 Prozent der Zweitstimmen liegen und eine der beiden Parteien den Löwenanteil der Direktmandate gewinnt, führen zu einer großen Anzahl von Überhangmandaten und zu einer entsprechenden Vergrößerung des Bundestags, die weit über der von 2013 liegen würde. Doch selbst diese stellte schon das größte relative Anwachsen des Bundestags gegenüber der regulären Sitzzahl in der Geschichte der Bundesrepublik dar, was von Norbert Lammert in seiner Antrittsrede kritisch bemerkt wurde.

\section{Fazit: alte Probleme behoben und neue geschaffen}

Ohne Zweifel bringt das neue Wahlgesetz in wesentlichen Punkten eine bedeutende Verbesserung gegenüber dem Gesetz von 2008. Dennoch fällt es nicht leicht, es zu preisen. Selbst wenn man die Schwierigkeiten betrachtet, die jeder Konsensfindung und dem damit einhergehenden Kompromiss zugrunde liegen, kann das neue Gesetz nicht als gelungen bezeichnet werden. Typischerweise sind Reformen von Wahlgesetzen durch machtpolitische Interessen einerseits und wertbezogene Ideen andererseits ${ }^{43}$ motiviert. Erstere scheinen dieses Mal - im Gegensatz zum Gesetz von 2011 - keine sonderliche Rolle gespielt zu haben. Das Design kann kaum den eng verstandenen Eigeninteressen der Parteien zu verdanken sein, denn das neue Gesetz schafft keiner Partei mehr einen systematischen und vorhersehbaren Vorteil. Genauso wenig Kann die Orientierung an theoretischen Ideen und normativen Konzepten erklären, warum das Wahlgesetz so ausgefallen ist. Es ist wenig wahrscheinlich, dass sich genau der vorliegende Inhalt ergeben hätte, wenn der Reformprozess als vorurteilslose und unbefangene Suche nach vernünftigen Lösungen für analytisch klar benannte Probleme abgelaufen wäre.

Tatsächlich lässt sich das Ergebnis wohl am besten als Konsequenz des historischen Entwicklungspfades erklären. Die Pfadabhängigkeit beruht dabei auf ganz normalen Zufälligkeiten und argumentativen Ermüdungserscheinungen, wie sie jedem langfristigen und

42 Vgl. Philipp Weinmann, a.a.O. (Fn. 34).

43 Vgl. Florian Grotz, a.a.O. (Fn. 3); zur komplexen Motivlage der an der Gesetzgebung Beteiligten vgl. auch Alan Renwick, The Politics of Electoral Reform, Cambridge 2010. 
kräftezehrenden Aushandlungsprozess zu eigen sind - wobei allerdings zwar keine machtpolitischen Interessen mehr hinsichtlich des Ergebnisses zu verteidigen waren, aber doch noch der eine oder andere symbolische Sieg zu erringen und vor allem der eine oder andere Gesichtsverlust noch zu vermeiden war. Letzteres gilt vor allem für diejenigen, die kurz zuvor mit ihrem Gesetz beim Bundesverfassungsgericht gescheitert waren. ${ }^{44}$

Die historische Pfadabhängigkeit des neuen Gesetzes lässt sich am stärksten an der Beibehaltung der ersten Verteilungsstufe erkennen, das heißt der anfänglichen Verteilung der Sitze auf Landessitzkontingente. Diese Komponente war schon im Entwurf von 2011 enthalten gewesen, allerdings mit den Wählerstimmen als Kriterium. Zweck der Konstruktion war die Beseitigung des negativen Stimmgewichts gewesen, ohne dabei die Überhangmandate anzugreifen. Mit der völlig veränderten Zieldefinition von Ende 2012 aber bestand eigentlich kein Anlass mehr, an diesem Element festzuhalten, da es seine ursprüngliche Funktion verloren hatte. In der Evolutionstheorie ist dieses Phänomen unter dem Begriff der „kulturellen Invarianz“ bekannt und manifestiert sich in dem Beibehalten inzwischen funktionslos gewordener Relikte. Das berühmte Beispiel von Konrad Lorenz bezieht sich auf die Gestaltung von Personenwagen der Eisenbahn im 19. Jahrhundert. ${ }^{45}$ Dabei orientierte man sich weiterhin an dem Design von Pferdekutschen, das heißt die ersten Personenwagen bestanden aus aneinandergesetzten Pferdewagen, die daher nur separat von außen bestiegen werden konnten. Es gab keinen durchgehenden, die Abteile verbindenden Gang, wie es die neu entstandenen Bedingungen der Eisenbahn sinnvoll gemacht hätten. Auf ganz analoge Weise verhindert beim Wahlrecht das Festhalten an einem schon einmal gescheiterten Entwurf, dass man den funktionellen Anforderungen der neu definierten Problemlage gerecht wird.

Genau wie eine gute wissenschaftliche Theorie muss sich ein gelungenes institutionelles Design gewissermaßen in den „Säurebädern von Experiment und Logik“" ${ }^{46}$ bewähren. Den argumentativen Herausforderungen genügt das neue Wahlsystem nur äußerst schwach. Mag es auch nicht verfassungswidrig sein, so ist es doch weit davon entfernt, weitergehende „legistische Desiderata ${ }^{“ 47}$ zu erfüllen wie Transparenz, Verständlichkeit und interne Kohärenz, um nur einige zu nennen.

Den Praxistest der letzten Bundestagswahl hat das neue Wahlgesetz ebenfalls nicht mit Bravour bestanden. In diesem natürlichen Experiment bewies sich unter noch relativ günstigen Umständen seine problematische Anfälligkeit für eine beachtliche Vergrößerung des Bundestags durch Ausgleichsmandate. Auch wenn dies noch nicht die von der Öffentlichkeit als problematisch eingestufte „Aufblähung“ ist und somit ein wirklich schwerwiegender "Unfall“ des Wahlsystems zunächst ausblieb, gibt es aber auch noch lange keinen Grund, jetzt untätig zu bleiben und alles so zu belassen, wie es ist. Beim neuen Wahlsystem besteht dringender Nachbesserungsbedarf, und es gibt Varianten, die den aufgetretenen Problemen gerecht werden und die nicht-intendierten Folgen vermeiden.

$44 \mathrm{Zu}$ diesen psychologischen Komponenten des Reformprozesses vgl. Hans Meyer, a.a.O. (Fn. 3); Heiko Holste, a.a.O. (Fn. 16).

45 Vgl. Konrad Lorenz, Die Rückseite des Spiegels, München 1973, S. 307 f.

46 Edward O. Wilson, Die Einheit des Wissens, Berlin 1998, S. 11.

47 Friedrich Pukelsheim / Matthias Rossi, a.a.O. (Fn. 3). 